\title{
Antioxidant factors, nitric oxide levels, and cellular damage in leprosy patients
}

\author{
Taysa Ribeiro Schalcher ${ }^{[1]}$, Jose Luiz Fernandes Vieira ${ }^{[1]}$, Claudio Guedes Salgado ${ }^{[2]}$, \\ Rosivaldo dos Santos Borges ${ }^{[1]}$ and Marta Chagas Monteiro ${ }^{[1]}$
}

[1]. Programa de Pós-Graduação em Ciências Farmacêuticas, Faculdade de Farmácia, Universidade Federal do Pará, Belém, PA. [2]. Programa de Pós-Graduação em Neurociências e Biologia Celular, Instituto de Ciências Biológicas, Universidade Federal do Pará, Belém, PA.

\begin{abstract}
Introduction: The immune response caused by Mycobacterium leprae is a risk factor for the development of oxidative stress (OS) in leprosy patients. This study aimed to assess OS in leprosy patients before the use of a multidrug therapy. Methods: We evaluated the nitric oxide (NO) concentration; antioxidant capacity; levels of malondialdehyde, methemoglobin and reduced glutathione; and the activity of catalase and superoxide dismutase (SOD) in leprosy patients. Results: We observed lower SOD activity in these leprosy patients; however, the NO levels and antioxidant capacity were increased. Conclusions: The infectious process in response to M. leprae could primarily be responsible for the OS observed in these patients.
\end{abstract}

Keywords: Mycobacterium leprae. Oxidative stress. Leprosy patients.

Leprosy, also known as Hansen's disease, is a chronic infectious disease caused by infection with the bacterium Mycobacterium leprae. This agent is an obligate intracellular bacillus that has a very low replication rate inside macrophages ( $\sim 13$ days) compared with the replication rates of other pathogenic mycobacteria, such as M. tuberculosis $(\sim 22 \mathrm{~h})^{1}$. Leprosy remains a significant public health issue in several parts of the world. In 2009 , more than 200,000 cases were registered worldwide by the World Health Organization (WHO), with approximately 40,000 of these cases occurring in Brazil ${ }^{1}$. Consequently, several additional epidemiological studies identified the main regions within Brazil that have a high prevalence of leprosy ${ }^{2}$.

With respect to the etiology of leprosy, several mechanisms for the resistance to the intracellular killing of $M$. leprae have been proposed, including the scavenging of free radicals produced by mononuclear phagocytes ${ }^{3}$. Microbial killing by macrophages is associated with a burst of respiratory activity that leads to the production of free radicals called reactive oxygen species (ROS), such as superoxide anions $\left(\mathrm{O}_{2}^{-}\right)$, hydrogen peroxide $\left(\mathrm{H}_{2} \mathrm{O}_{2}\right)$, hydroxyl radicals and reactive nitrogen species (RNS), including nitric oxide $(\mathrm{NO})^{4}$. The human body has developed endogenous antioxidant systems to detoxify the body of free radicals as required ${ }^{4}$. Oxidative stress (OS) is an expression used to describe the various deleterious processes resulting from an imbalance between the free radical-generating and free radicalscavenging systems. OS leads to metabolic impairment and cell

Address to: Dra. Marta Chagas Monteiro. PPGCF/Lab Microbiologia e Imunologia Clínica/FF/UFPA. Av. Augusto Correa s/n, Guamá, 66075-110 Belém, PA, Brasil.

Phone: 5591 3201-7202; Fax: 5591 3201-7201

e-mail: martachagas2@yahoo.com.br

Received 3 January 2012

Accepted 19 April 2012 death, and it occurs when ROS are not adequately scavenged by antioxidants ${ }^{4}$. A decrease in the antioxidant status can contribute to an increase in OS and thereby complicate the treatments and control mechanisms available for these patients. The aim of this study was to determine the OS levels in leprosy patients before initiating the use of a multidrug therapy by estimating the levels of the antioxidant agents and $\mathrm{NO}$ in addition to products generated by OS, such as lipid peroxidation products, malondialdehyde (MDA) and methemoglobin (MetHb).

Twenty-three newly diagnosed leprosy patients that attended the outpatient department at the State Reference Unit for Leprosy Treatment - Dr. Marcello Candia, Marituba and the Health Unit Guamá, Belém, PA, Brazil between May 2010 and May 2011 were selected for the study prior to starting a multidrug therapy regiment. The patients, with an age range of 20-45 years, were classified into a paucibacillary (PB) group (5 cases) and a multibacillary (MB) group (18 cases) based on the WHO guidelines ${ }^{1}$. The diagnoses were performed on clinical grounds by using slit-skin smear tests. Leprosy patients with reactions, ulcerations, a history of smoking, co-infections, diabetes mellitus, other systemic diseases or health problems or a history of drug use including vitamins, ascorbic acid or tocopherol in addition to those under the influence of alcohol or over 45 years of age were excluded to rule out any possible influence of these confounders. Healthy adult volunteers were selected to serve as controls (20 individuals) for the study. The control group did not have signs or symptoms of leprosy, other systemic diseases, or health problems. Additionally, the volunteers in the control group were non-smokers and free from drug use. The control group consisted of healthy, sex-matched individuals aged 20-45 years who were living in the same settings as the leprosy patients. The Ethical Committee of the Federal University of Pará approved the study protocol (protocol 079/09). All participants signed a committee-approved consent 
form. The diagnosis of leprosy was based on the WHO clinical criteria (testing positive for 2 of the 3 clinical criteria, which are skin lesions, anesthesia and nerve enlargement). The patients were classified clinically as having either $\mathrm{PB}$ or MB leprosy based on the number of skin lesions $(\leq 5$ lesions $=\mathrm{PB}$ and $>5$ lesions $=\mathrm{MB})$. The bacterial index (BI) was also calculated ${ }^{1}$.

Blood samples were obtained from all patients by venipuncture in tubes containing ethylenediaminetetraacetic acid (EDTA). A portion of the whole blood was used immediately for determining the levels of MetHb and reduced glutathione (GSH) in addition to the activity of superoxide dismutase (SOD), whereas the additional blood was centrifuged at $2,000 \times \mathrm{g}$ for 6 minutes to separate the plasma for the analyses of thiobarbituric acid-reactive substances (TBARS) and Trolox equivalent antioxidant capacity (TEAC). In addition, the serum was also collected for NO measurement. The MetHb content was measured in duplicate by an absorbance reading at a wavelength of $632 \mathrm{~nm}$. Values less than $2 \%$ were considered normal. Lipid peroxidation was measured by the quantification of MDA using TBARS as an estimation. This method is a very useful, inexpensive and easy-to-use assay for the evaluation of $\mathrm{OS}^{5}$. Briefly, lipoproteins were precipitated from the samples by the addition of trichloroacetic acid (TCA) (to a final concentration of $0.05 \mathrm{M}$ ) and $0.7 \%$ thiobarbituric acid (TBA) in $2 \mathrm{M}$ sodium sulfate. The lipid peroxide and TBA reaction was performed by heating the mixture in a water bath for $90 \mathrm{~min}$. The resulting chromogen that was formed was extracted in n-butanol and measured at a wavelength of $535 \mathrm{~nm}$. Lipid peroxidation was expressed as nanomoles of MDA per liter.

In this study, the antioxidant capacity of the samples was determined by measuring the TEAC using a calibration curve plotted against different amounts of Trolox in absorbance units measured at a wavelength of $740 \mathrm{~nm}$. This method is based on the prevention of persulfate oxidation of 2,2'-azino-bis (3-ethylbenzothiazoline-6-sulphonic acid) (ABTS) to ABTS ${ }^{2-}$ by the antioxidants present in the sample, to a degree proportional to the concentration of the antioxidants. Moreover, OS was estimated using the MetHb/GSH, MDA/GSH, MDA/SOD and $\mathrm{NO} / \mathrm{SOD}$ ratios. These ratios are affected by the antioxidant response against pro-oxidant production in an organism, with higher values indicating a higher OS.

The nitrate $\left(\mathrm{NO}_{3}^{-}\right)$present in the patient serum samples was reduced to nitrite using nitrate reductase, and the nitrite concentration was determined by the Griess method. The absorbance was then measured at $550 \mathrm{~nm}$ to determine the nitrite $\left(\mathrm{NO}_{2}-\right)$ concentration (in millimoles). The intracellular GSH levels (in $\mu \mathrm{mol} / \mathrm{mL}$ ) were quantified by measuring the absorbance at $412 \mathrm{~nm}$. The level of SOD activity (in $\mathrm{nmol} / \mathrm{mL}$ ) was quantified by measuring the absorbance at a wavelength of $550 \mathrm{~nm}$. CAT values were expressed as units per gram of hemoglobin (U/g protein), and the decay of $\mathrm{H}_{2} \mathrm{O}_{2}$ was measured at $240 \mathrm{~nm}$. Data are reported as the mean $\pm \mathrm{SD}$ and statistically significant differences between the groups were determined using Student's $t$-test $(\mathrm{p}<0.05)$. Pearson's coefficients were used to determine correlations among the concentrations of $\mathrm{MetHb} / \mathrm{GSH}, \mathrm{MDA} / \mathrm{GSH}, \mathrm{MDA} / \mathrm{SOD}$ and NO/SOD in the blood samples.
Most patients were excluded from the study because of the strict patient selection criteria; however, 23 enrolled patients aged 20-45 years were diagnosed with leprosy and met the remaining inclusion criteria. The final study cohort comprised these 23 patients and 20 control subjects. Of these patients, $22 \%$ were classified as $\mathrm{PB}(\mathrm{n}=5)$ and $78 \%$ as $\mathrm{MB}(\mathrm{n}=18)$. The ages of the patients in the PB group varied from 23 to 45 years, with a mean of $35.2 \pm 4.1$ years. The ages in the MB group varied from 20 to 45 years, with a mean of $29.8 \pm 2.1$ years. The age of the control group varied from 20 to 45 years, with a mean of $27.5 \pm 2.4$ years. Of the $18 \mathrm{MB}$ patients, $6(33 \%)$ had a positive

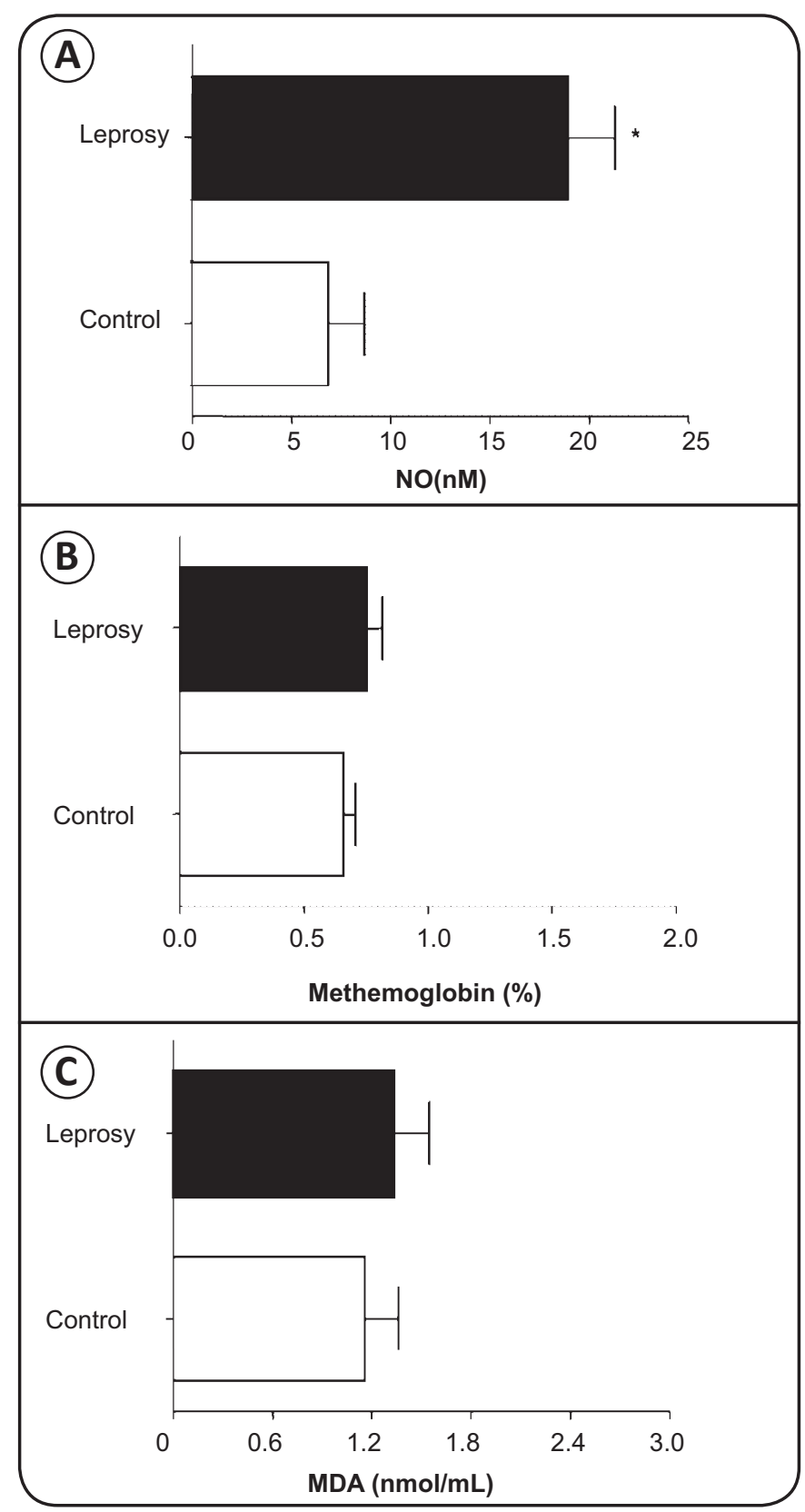

FIGURE 1 - A: Determination of nitric oxide in serum. B: Fraction of methemoglobin in the blood. C: Levels of malondialdehyde in the plasma of patients with untreated leprosy. Data are presented as the mean \pm SEM; $* \mathrm{p}<0.05$ compared with controls ( $t$-test). NO: nitric oxide, MDA: malondialdehyde. 
TABLE 1 - Ratios between oxidant (MetHb, MDA and NO) and antioxidant agents (GSH and SOD) from leprosy patients and control individuals.

\begin{tabular}{|c|c|c|c|}
\hline Variable & Control & Leprosy & $\mathrm{p}$ value \\
\hline MetHb/GSH & $0.19 \pm 0.07$ & $0.47 \pm 0.49 *$ & $\mathrm{p}<0.001$ \\
\hline MDA/GSH & $0.34 \pm 0.13$ & $0.18 \pm 0.23 *$ & $\mathrm{p}<0.001$ \\
\hline MDA/SOD & $0.007 \pm 0.006$ & $0.025 \pm 0.005 *$ & $\mathrm{p}<0.05$ \\
\hline $\mathrm{NO} / \mathrm{SOD}$ & $0.05 \pm 0.07$ & $0.27 \pm 0.08 *$ & $\mathrm{p}<0.05$ \\
\hline
\end{tabular}

MetHB: methemoglobin; GSH: reduced glutathione; MDA: malondialdehyde; SOD: superoxide dismutase; NO: nitric oxide. *p: significance level; values were expressed as mean \pm standard deviation.

bacterial index (BI) varying from 1 to 6,7 had various degrees of disability (scores of 1 or 2 ) and 5 had nerve damage (scores of 1 to 3). None of the PB patients displayed a positive BI, any degree of disability or nerve damage.

In addition, the NO levels were significantly increased in the leprosy patients compared with the control individuals (Figure 1A). The MDA values and MetHb percentages observed in the leprosy patients did not differ from those in the control group (Figures 1B and 1C). The mean value of erythrocyte SOD activity was significantly lower in the leprosy patients than in the control group (Figure 2A). The GSH levels and CAT activity in leprosy patients were similar to those in the control group (Figures 2B and 2C, respectively). In addition, there was a significant increase in the mean values of the blood TEAC in the leprosy patients compared with the control group (Figure 2D). Table 1 illustrates the ratios of the oxidant and antioxidant levels in samples from the leprosy patients and control individuals. Our data showed significant differences in the $\mathrm{MetHb} / \mathrm{GSH}, \mathrm{MDA} / \mathrm{GSH}, \mathrm{MDA} / \mathrm{SOD}$ and NO/SOD ratios of the leprosy patients compared with the control group and thus

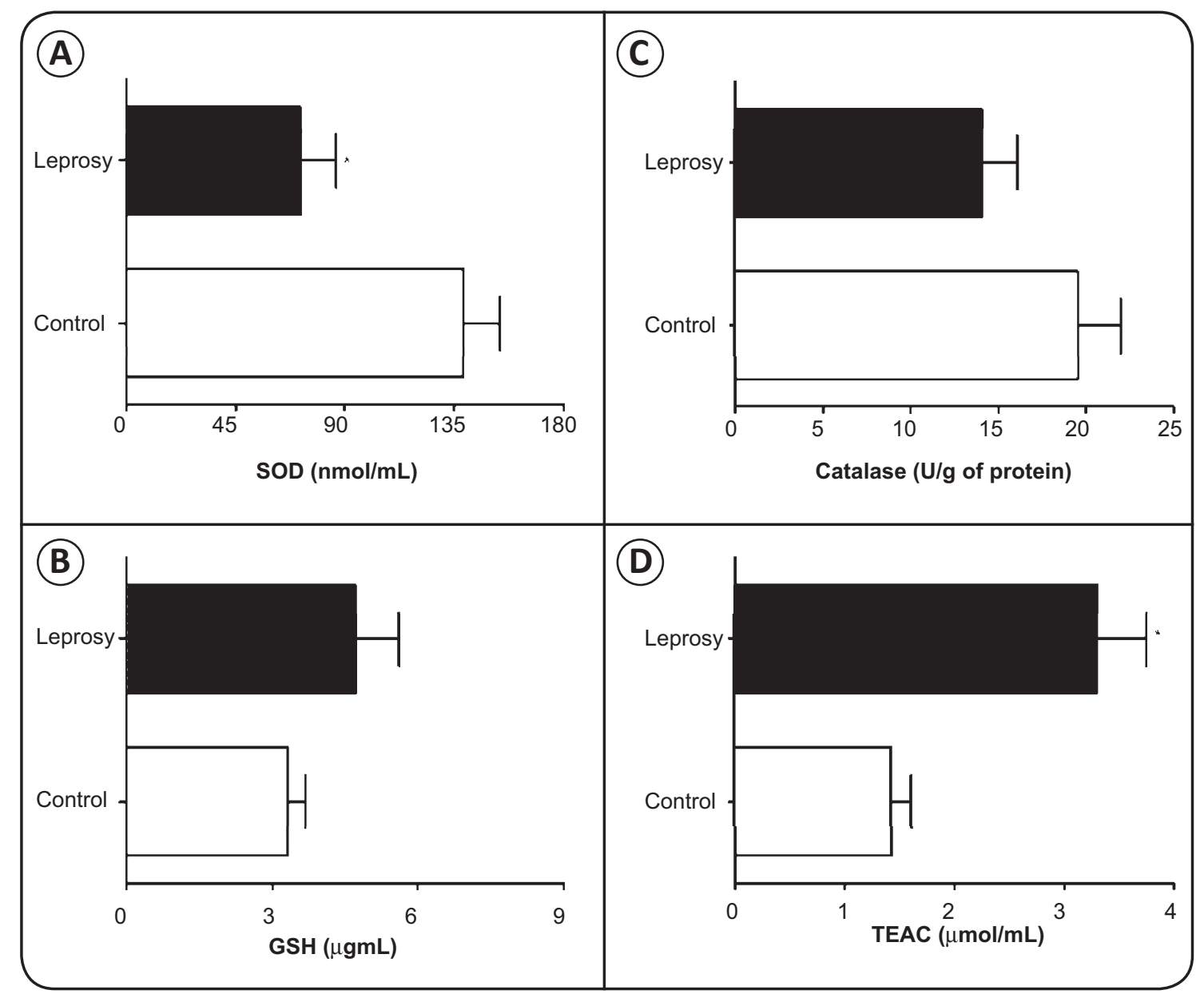

FIGURE 2 - Antioxidant capacity of blood samples from untreated leprosy patients. A: Concentration of superoxide dismutase. B: Reduced glutathione. C: Catalase. D: TEAC. Data are presented as the mean \pm SEM; $* p<0.05$ compared with controls $(t$-test). GSH: reduced glutathione; SOD: superoxide dismutase; TEAC: trolox equivalent antioxidant capacity. 
represent an index of OS that causes metabolic impairments. Additionally, the cell death values of the leprosy patients were different from those of the control group.

The NO values significantly increased in the leprosy patients compared with the control group. Other studies also demonstrated increased $\mathrm{NO}$ synthesis or expression of inducible NO synthase (iNOS) in macrophage-rich granulomas and peripheral nerves in leprosy patients, which indicates that NO is involved in nerve damage and the elimination of infectious agents ${ }^{6}$. Schön et al. ${ }^{6}$ demonstrated the presence of reactive species, such as $\mathrm{NO}$ and peroxynitrite ( $\left.\mathrm{ONOO}^{-}\right)$, produced by macrophages in the skin lesions of patients with borderline forms of leprosy, which suggests that these species could be related to the nerve damage noted in these patients.

An increased serum/plasma MDA level is regarded as a standard biochemical marker of the lipid peroxidation and cellular damage caused by free radicals ${ }^{5}$; however, some studies have shown no significant changes in the MDA levels of leprosy patients ${ }^{7,8}$. These findings are similar to our results showing that MDA levels in leprosy patients remain unchanged. In addition, we also observed that the fraction of MetHb in samples from patients who did not receive MDT treatment was not altered relative to the control group. Notably, these MetHb levels were within the normal limits $(<2 \%)$. These data are similar to those reported by Rimiolli and Godoy ${ }^{9}$, where non-treated MB leprosy patients also had normal MetHb levels.

To eliminate free radicals and thereby minimize tissue injury, several antioxidant enzymes, such as SOD, CAT and GPx, scavenge the ROS accumulated in the cells ${ }^{4,10}$. In this study, leprosy patients presented significant decreases in SOD activity but not CAT and GSH levels. Moreover, the TEAC in these patients was higher than that in the control group. Other studies obtained findings similar to ours. Abdel-Hafez et al. ${ }^{11}$ also observed that SOD levels were significantly lower in MB and PB leprosy patients than in the control group. SOD is one of the major antioxidant enzymes that scavenges free radicals. It acts as an endogenous cellular defense system that converts $\mathrm{O}_{2}^{-}$to $\mathrm{H}_{2} \mathrm{O}_{2}$ and oxygen ${ }^{11}$, with the latter being further detoxified by CAT and GPx. Additionally, nutritional antioxidants also trap free radicals and act as free radical-scavenging systems ${ }^{10}$. The low SOD activity observed in our study may be associated with higher ROS levels caused by the $M$. leprae-induced immune response. This effect may be related to the antigen-binding property of Hansen's bacillus, such as the binding of phenolic glycolipid 1 (PGL-1) to the SOD enzyme, which blocks SOD activity or down-regulates SOD gene expression in red blood cells and macrophages ${ }^{12}$. Another hypothesis that may explain the decreased levels of SOD in the leprosy patients in our study is the lack of consumption of intracellular metals, such as zinc, by these patients. These metals are needed for the survival and multiplication of $M$. leprae. As a bystander effect, the lack of these metals may also act negatively on the activity of SOD, which requires metals such as copper and zinc for its enzymatic activity ${ }^{13}$.

As observed in this study, the TEAC level was elevated in the plasma of the leprosy patients, and this increase could be associated with the type of sample evaluated. In plasma,
TEAC increases have been attributed to low-molecular-weight antioxidants from food sources, whereas TEAC determination in cells is mainly associated with enzymatic antioxidant systems such as SOD and $\mathrm{CAT}^{14}$. In addition, many proteins such as ceruloplasmin, transferrin and small antioxidant molecules such as non-protein thiols, vitamins $\mathrm{C}$ and $\mathrm{E}$ and uric acid account for the remainder of the plasma TEAC ${ }^{15}$. These data suggest that the increase in plasma TEAC in leprosy patients could therefore result from elevated levels of antioxidant molecules, protein thiols or non-thiols. In summary, we observed a decrease in the levels of enzymatic antioxidants such as SOD and an increase in the levels of $\mathrm{NO}$ in leprosy patients, which suggests that the pathogenesis caused by $M$. leprae infection in these patients included a systemic oxidant/antioxidant imbalance.

\section{ACKNOWLEDGMENTS}

We are grateful to State Reference Unit for Leprosy Treatment Dr. Marcello Candia, Marituba and the Health unit Guamá, for giving us space for this research. We are also grateful to the toxicology laboratory of the Federal University of Pará, where we performed most of the experiments.

\section{CONFLICT OF INTEREST}

The authors declare that there is no conflict of interest.

\section{FINANCIAL SUPPORT}

Conselho Nacional de Pesquisa (CNPq), Fundação Amazônia Paraense de Amparo à pesquisa (FAPESPA) and Federal University of Pará, Brazil.

\section{REFERENCES}

1. World Health Organization. Global Leprosy Situation.Wkly Epidemiol Rec 2009; 33:333-340.

2. Sanches LAT, Pittner E, Sanches HM, Monteiro MC. Detecção de casos novos de hanseníase no município de Prudentópolis, PR: 1998 a 2005. Rev Soc Bras Med Trop 2007; 40:541-545.

3. Kang TJ, Chae GT. Detection of Toll-like receptor 2 (TLR2) mutation in the lepromatous leprosy patients. FEMS Immunol Med Microbiol 2001; 31:53-58

4. Valko M, Leibfritz D, Moncol J, Cronin MT, Mazur M, Telser J. Free radicals and antioxidants in normal physiological functions and human disease. Int J Biochem Cell Biol 2007; 39:44-84.

5. Bickers DR, Athar M. Oxidative stress in the pathogenesis of skin disease. J Invest Dermatol 2006; 126:2565-2575.

6. Schön T, Hernandez-Pando RH, Negesse Y, Leekassa R, Sundqvist T, Britton S. Expression of inducible nitric oxide synthase and nitrotyrosine in borderline leprosy lesions. Br J Dermatol 2001; 145:809-815.

7. Reddy YN, Murthy SV, Krishna DR, Prabhakar MC. Oxidative stress and anti-oxidant status in leprosy patients. Indian J Lepr 2003; 75:307-316.

8. Prasad CVB, Kodliwadmath MV, Kodliwadmath GB. Erythrocyte superoxide dismutase, catalase activities and hydrogen peroxide induced lipid peroxidation in leprosy. Lepr Rev 2007; 78:391-397.

9. Rimiolli LF, Godoy MF. Quantificação do estresse oxidativo no sangue de hansenianos sob efeito ou não de tratamento específico. Hansenol Int 2001; 26:93-98. 
10. Pinnell SR. Cutaneous photodamage, oxidative stress, and topical antioxidant protection. J Am Acad Dermatol 2003; 48:20-22.

11. Abdel-Hafez HZ, Mohamed EE, Abd-Elghany AA Tissue and blood superoxide dismutase activity and malondialdehyde level in leprosy. J Eur Acad Dermatol Venereol 2010; 24:704-708.

12. Bhadwat VR, Borade VB. Increased lipid peroxidation in lepromatous leprosy. Indian J Dermatol Venereol Leprol 2000; 66:121-125.

13. Jain A, Mukherjee A, Chattopadhya D, Saha K. Biometals in skin and sera of leprosy patients and their correlation to trace element contents of
M. leprae and histological types of the disease; a comparative study with cutaneous tuberculosis. Int J Lepr Other Mycobact Dis 1995; 63:249-258.

14. Ghiselli A, Serafini M, Natella F, Scaccini C. Total antioxidant capacity as a tool to assess redox status: critical view and experimental data. Free Radic Biol Med 2000; 29:1106-1114.

15. Rahman I, Swarska E, Henry M, Stolk J, MacNee W. Is there any relationship between plasma antioxidant capacity and lung function in smokers and in patients with chronic obstructive pulmonary disease? Thorax 2000; 55:189-193. 